\title{
Adaptability of Distribution Automation System to Electric Power Quality Monitoring In Nigeria Power Distribution Network
}

\author{
Oyetunji S.A. \\ Department of Electrical/Electronic Engineering, Federal University of Technology, Akure, Nigeria
}

\begin{abstract}
The trend in Nigeria is shifting from providing electricity to consumers only but with improved quality as a result of deregulation. This paper demonstrates the adaptability of distribution automation system to electric power quality on-line monitoring using Nigeria distribution system as a case study. This paper reviews power quality problems, electric power distribution topology and monitoring quantities. The paper explains the development of program for the analysis of power quality. Sensors were incorporated at the substations for the measurement of substation voltages and line currents. The embedded system coordinates the activities of these sensors and initiates communication at regular intervals with the central server for analysis. The data back-up was implemented on external card which are available for down load on monthly basis. The results reveal neglect to the issues of quality, failure to comply with regulations in some of the substations investigated. The study is expected to benefit the operator in the area of planning and improved power delivery.
\end{abstract}

Keywords: Current, Distribution Automation, Power Quality and Voltage

\section{Introduction}

Over the year Nigeria as a developing country has being faced with the challenges of meeting the energy need of the consumers. However, this increase in power supply infrastructures is without strict compliance to the issue of quality. Power quality can be defined from two different perspectives, consumer or supplier perspectives. The definition of power quality becomes even more unclear when the roles of utility and the customer become blurred as the utility industry is restructured and deregulated. This study becomes important particularly as it affects the next phase of power industry in Nigeria-deregulation. Several methods for detection and classification of PQ disturbances have been published. Some of them focus only on one particular type of disturbance [1], others aim to cover a wider range of disturbances [2]. The wavelet transform [3] is one of the most often employed signal processing algorithms [4]. It has been applied for detection of transients as well as sags or swells. However in the latter case it exhibits several drawbacks arising from weak response to sags and swells of a certain shape (especially when the voltage drops and increases are not sudden but gradual). Power quality disturbances that may occur in a power system can be extremely different in their characteristics such as magnitude, duration and wave-shape. In accordance with [5] only one technique might not be suitable to capture the PQ disturbance characteristics presented by various disturbances on the electric power distribution system. However based on these characteristics, the nature of disturbances can be classified into two categories which are magnitude and wave-shape and Duration. The proposed algorithm is based on assessing the quality of the power supply using the magnitude and duration. Duration variations represent category of PQ disturbances detected by analyzing the RMS value of the normalized input voltage. In the classification stage, the disturbances are classified based on their magnitude and duration.

\subsection{Types of Power Quality Problems}

According to [6], power quality problems contain several items such as interruptions, under voltage, over voltage, lightning and switching surges, sags, swells, harmonics, voltage unbalance, flicker and so on. The various components that constitute power quality problems are discussed as follows $[7,8]$ :

\subsubsection{Voltage sags (dips)}

IEEE defines voltage sags as a reduction in voltage for a short time and are referred to voltage dips. The duration of voltage sag is less than 1 minute but more than 10 milliseconds ( 0.5 cycle). The magnitude of the reduction is between 10 percent and 90 percent of the normal root mean square (rms) voltage at $50 \mathrm{~Hz}$.

\subsubsection{Voltage swells}

Voltage swells, or momentary over-voltages, are rms voltage variations that exceed 110 percent of the nominal voltage and last for less than 1 minute. Voltage swells occur less frequently than voltage sags. Singleline to ground faults causes voltage swells. Long-duration over voltages are voltage swells that last longer. 
1.1.3 Under voltage

Under-voltages occur when the voltage drops below 90 percent of the nominal voltage for more than 1 minute. They are recognized by end users when their lights dim and their motors slow down. . This is typical of most stations visited in Akure Distribution Network.

\subsubsection{Interruptions}

Interruptions are a complete loss of voltage (a drop to less than 10 percent of nominal voltage) in one or more phases. IEEE defines three types of interruptions. They are categorized by the time that the interruptions occur: momentary, temporary, and long-duration interruptions. Momentary interruptions are the complete loss of voltage on one or more phase conductors for a time period between 0.5 cycles, or 8 milliseconds, and 3 seconds. A temporary, or short-duration, interruption is a drop of voltage below 10 percent of the nominal voltage for a time period between 3 seconds and 1 minute. Long-duration, or sustained, interruptions last longer than 1 minute. Long-duration interruption affects the consumers and receives attention by the operators in the distribution network investigated

\subsubsection{Voltage unbalance}

Voltage unbalance is the deviation of each phase from the average voltage of all three phases. It can be calculated that voltage unbalance ratio equals maximum deviation from average voltage divided by average voltage multiples $100(\%)$.

\subsubsection{Voltage fluctuations}

Voltage fluctuations are rapid changes in voltage within the allowable limits of voltage magnitude of 0.95 to 1.05 of nominal voltage. Voltage fluctuations can cause incandescent and fluorescent lights to blink rapidly. This blinking of lights is often referred to "flicker." This change in light intensity occurs at frequencies of 6 to $8 \mathrm{~Hz}$ and is visible to the human eye.

\subsubsection{Harmonics}

Harmonics are the major source of sine waveform distortion. The increased uses of nonlinear equipment have caused harmonics to become more common. Harmonics are integral multiples of the fundamental frequency of the sine wave, that is, harmonics are multiples of the $50 \mathrm{~Hz}$ fundamental voltage and current. They add to the fundamental $50 \mathrm{~Hz}$ waveform and distort it. Harmonics are usually caused by nonlinear loads. The THD can be used to characterize distortion in both current and voltage waves.

\subsection{Real-time Monitoring of PQ}

Effective monitoring of the power quality is only visible with distribution system automation system (DAS)[8]. However, real-time data on the conditions of distribution substations are currently not available for remote diagnosis in Nigeria. For example, the information on the loading of distribution transformer substations is not in real time, but is limited and based only on technical checks performed few times a year by visiting maintenance crew. Due to the vast number of units to be monitored, utilities find the implementation of communication between numerous monitoring systems very expensive to install and maintain and as such have tended to ignore this aspect entirely. However, recent advances in wireless communication technology especially the mobile phone networks [9] (NCC 2009), offer new, cost effective possibilities for monitoring otherwise impossibly complex EPDS. The wide spread use of global system for mobile communication (GSM) [9] with provision for short message service offers a possible solution for linking between remotely located distribution equipment and the control center.

\subsubsection{Development of DAS}

The distribution can be categorised into three levels based on the European standard IEC-61850 (IEC2003) [10]. IEC-61850 is a superset of Architecture Utility Communication UCA 2.0 (Clinard2002) as expressed in Figure 1.

- Process level

- Bay level and

- Station Level

The process level consists of the field instrumentation for monitoring the parameters of the network both at the substation and on the overhead feeders. The signal transmitted to the bay level for logging and diagnosis. The RTU operates at the bay level. The bay level communicates with the station level when a change is detected in the parameters values outside the defined threshold. The station level carries out analysis and diagnosis. Figure 2.12 shows the standard DAS [10 ], [11] and [12]. 


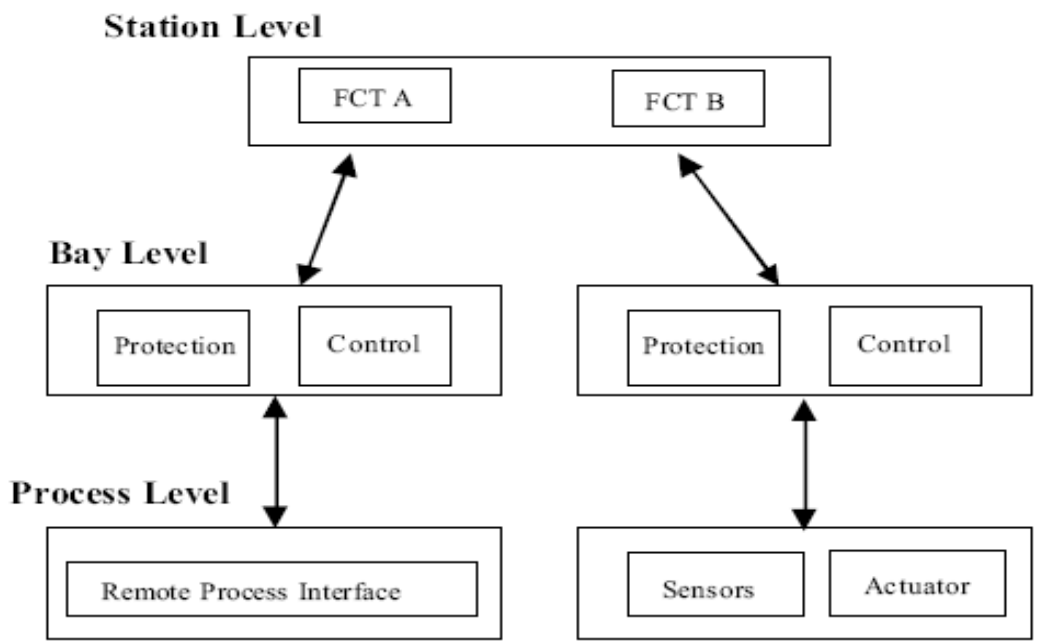

Figure 1: European standard for distribution automation system (IEC-61850)

\subsection{Communication System for Real-Time Monitoring}

Perhaps, the most difficult task in automating a distribution network is the selections of an appropriate communications technique for the implementation of remote control/reporting facilities [13 ] (Gupta 2008). According to $[14,15,16]$ several techniques are available, as shown Figure 2.14 to establish communication between the RTU and DCC. These are:

a) hard-wired

b) Public Switched Telephone Network (PSTN)

c) mobile radio (packet switched data)

d) conventional or low-powered radio (includingMicrowave)

e) Power Line Carrier Communication (PLCC)

\subsubsection{Wireless communication: Global System for Mobile Communication}

Global system for mobile communication (GSM) has grown considerably since its introduction in 1990. In Nigeria, three GSM operators which commenced operation in 2001 with less than 1 million subscribers have since attained a subscriber base of over 50 million by 2009. The GSM operators provide voice, data and Short Message Service (SMS) for subscribers. The SMS possesses some advantages when utilized as a communication interface for distribution automation. According to [9] (NCC2009) the five Operators licensed to provide mobile GSM services in Nigeria are ( Figure 2):

- MTN Nigeria Communications Limited

- Airtel Nigeria Limited

- Glo Mobile Nigeria Limited

- Nigerian Mobile Telecommunications Limited (M-tel)

- Emerging Markets Telecommunications Services Limited (Etisalat)

Other Telecoms providers include operators on fixed wired/wireless and CDMA platform.

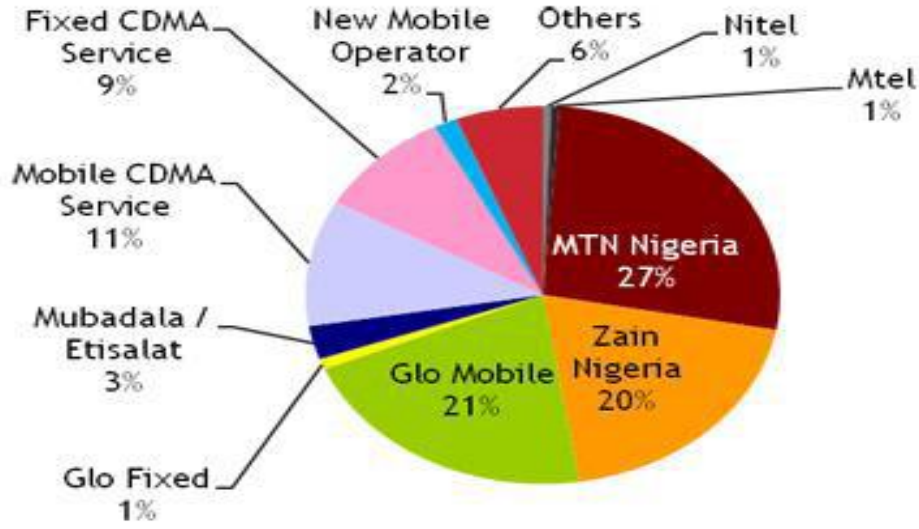

Figure 2: Telecommunication providers' distribution in Nigeria 
Table 1: GSM growth in Nigeria-telecoms subscriber information (2001- 2008)

\begin{tabular}{|l|l|l|l|l|l|l|l|l|}
\hline $\begin{array}{l}\text { OPER } \\
\text { ATOR }\end{array}$ & 2001 & 2002 & 2003 & 2004 & 2005 & 2006 & 2007 & 2008 \\
\hline GSM & 266,461 & $1,569,050$ & $3,149,472$ & $9,174,209$ & $18,295,896$ & $32,184,861$ & N/A & N/A \\
\hline $\begin{array}{l}\text { Telede } \\
\text { nsity } \\
{[1]}\end{array}$ & $0.73]$ & 1.89 & 3.35 & 8.50 & 16.27 & 24.18 & 29.9 & 45.93 \\
\end{tabular}

\section{Remote Measurements}

According to [17] (Tenbohlen 2000) remote measurements can be obtained in three different ways:

a) Cyclic polling,

b) Polling on demand, or

c) Threshold trespassing.

Threshold trespassing was implemented which supports the Knowledge based algorithm. The cyclic polling requires ubiquitous network which is expensive to operate in the environment under consideration using Mobile communication. Similarly, polling on demand involves two way communications which translates to additional transfer cost.

The indigenous concept based on European Protocol as shown in Figure 1 was developed for Akure EPDS. The block diagram in Figure 3 shows the processes involved in achieving the stated objectives. The instrumentation units reside in the process level while the substation remote terminal unit formed the bay level and the station distribution central controller constitute the station level. For connecting the process level to the bay level a Star topology LAN was chosen.

RTU transfers this information to master station DCC using DNP3.0 directly [1 ]. RTU has the following main functions:

a) Communication between host and Instrumentation units;

b) Measuring of analog data (I, V);

c) Function of saving historical data; and

d) Communication between the RTU and the DCC.

The DCC acts as the master controller and resides at the central office. The function includes

a) Receive data from RTU;

b) Data Analysis;

c) Function of saving historical data;

d) Display on GUI;

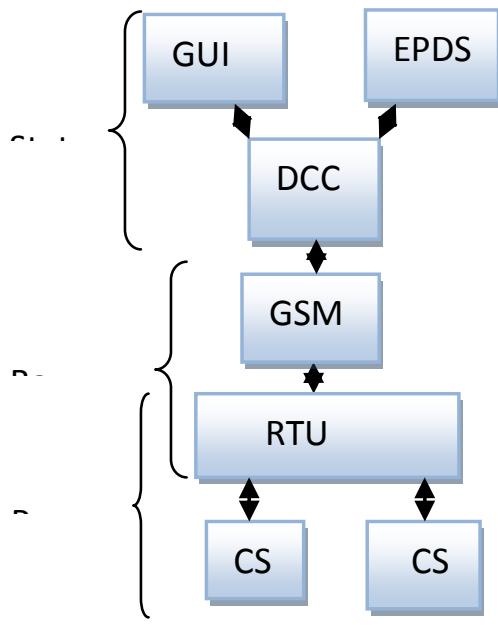

Figure 3: Conceptual distribution network remote monitoring model

\section{RTU for PQ Monitoring}

The RTU is designed to response to changes associated with the two parameters. The RTU utilizes the power quality function and the current signatures to determine the state of the network. If RTU detects bad power quality, it initiates the data acquisition of the three phase voltage and current and sends the information immediately to the DCC. In general, RTU collects analog data such as current and voltage value of three phases and transfers the digital data. 


\section{Arithmetic Using Microprocessor}

Three phase voltages and currents are fed to RTU from Current Instrumentations and PTs at the substation's terminal of the feeder. These voltages and currents are instantaneous values, and the effective value was made from this instantaneous value using RMS arithmetic method at RTU.

$$
V_{r m s}=\sqrt{\frac{1}{N}} \sum_{i=0}^{N} V_{i}^{2}
$$

The THD is calculated as

$$
V_{T H D}=\frac{\sqrt{\sum_{n \geq 2} V_{n}^{2}}}{V_{1}} X 100 \%
$$

Voltage Unbalance calculated as

$$
\frac{V_{n}-V_{r m s}}{V_{n}} X 100 \%
$$

Where $V_{n}$ is the nominal voltage and $V_{r m s}$ is the recorded phase voltage

\section{Experimentation Set Up} following:

The field implementation was developed as shown in Figure 5. The measuring set-up consists of the

(i) sensor box which includes voltage and current transducers that measure the voltage and current in the power system;

(ii) data acquisition board (DAQ) which acquires the signals from the transducers;

(iii) PC which controls the data acquisition process and performs the signal processing required by the proposed method. An important part of the measuring set-up is the uninterruptible power supply (UPS) which secures power for the PC and the transducers during long disturbances (especially in case of interruptions).

The sensor box contains a voltage transducer and a current transducer. The data acquisition board was built on PIC microcontroller with 10 bit resolution and sampling frequency up to $100 \mathrm{kS} / \mathrm{s}$. The developed software includes a measuring and detection program which continuously acquires data from the sensor box, analyses them and stores information about the detected and classified disturbances. The described measuring set-up was used for long-term monitoring of single-phase power system $230 \mathrm{~V} / 50 \mathrm{~Hz}$. The set-up was installed in Federal university of technology Akure, Nigeria where it gathered data during 3 months. The detected PQ disturbances were classified and information, including the relevant voltage waveforms, were stored in the PC.

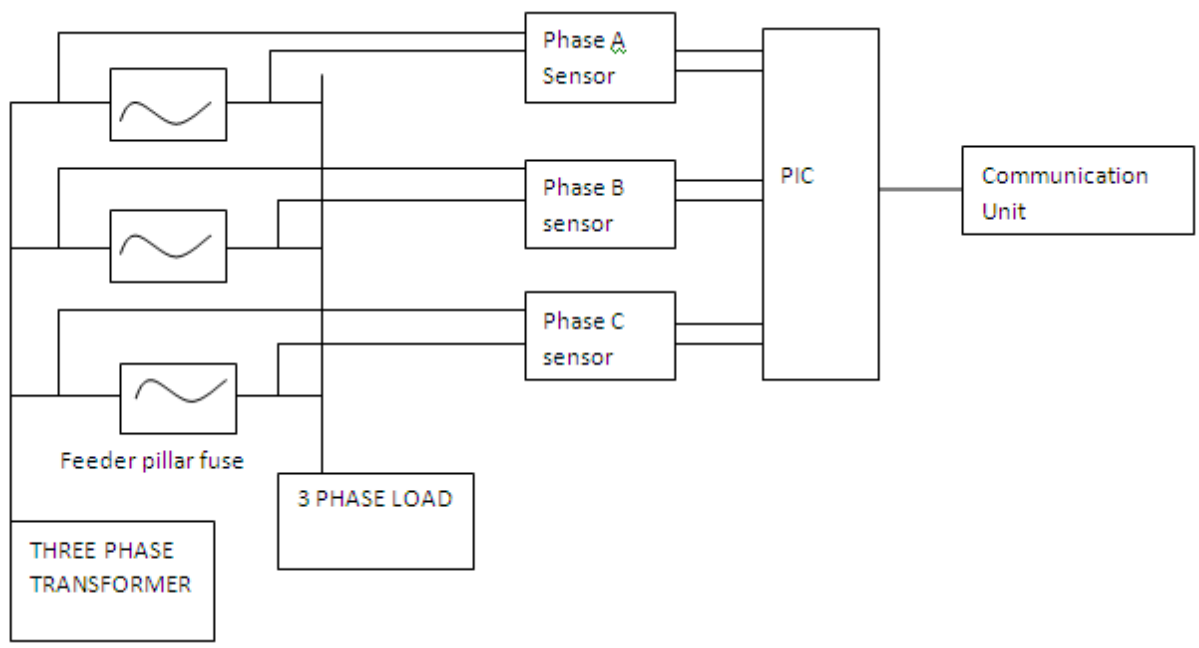

Fig. 5. Measuring Set-Up for Power Quality Monitoring.

\section{Diagnosis}

At the DCC, the data is received through a mobile phone connected to the processing PC using developed $\mathrm{C}$ \#based software. The two platforms that provided tools for data analysis include MATLAB and Engineering software. 
The waveforms for current and voltage for each phase are captured and displaced as shown in Fig. 6. The Engineering software provides information on the quality of the power supply based on the data received. Some of the windows based on the received data are shown in Fig. 7

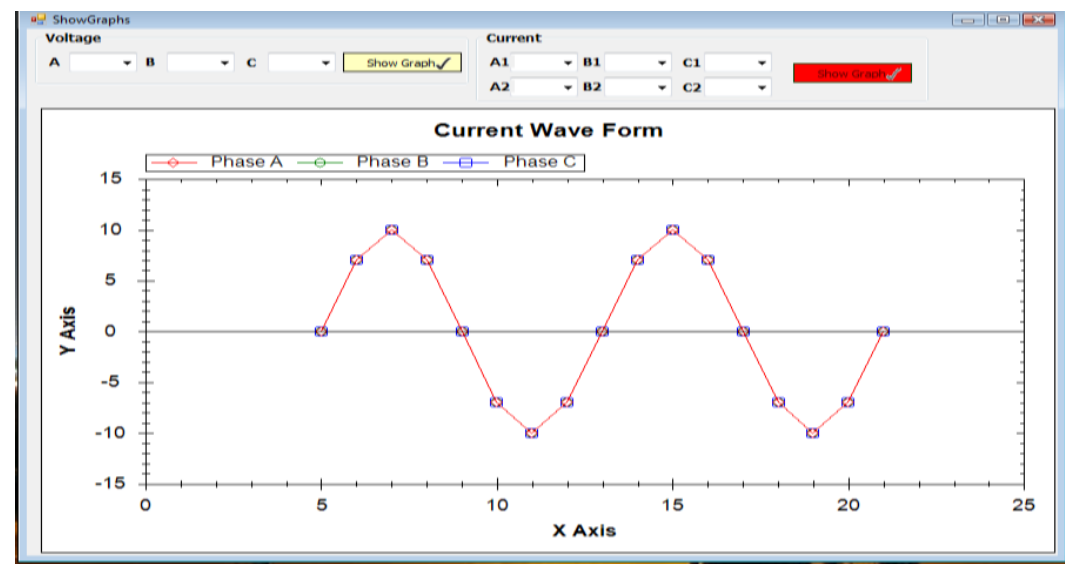

Fig. 6. Analysis of measured PQ disturbances.

Received Data for interruption:

\$1001737374000000161D2614080000000000000000000000000000000C120C000F160F000D130D00141D14 000D130D001A261A

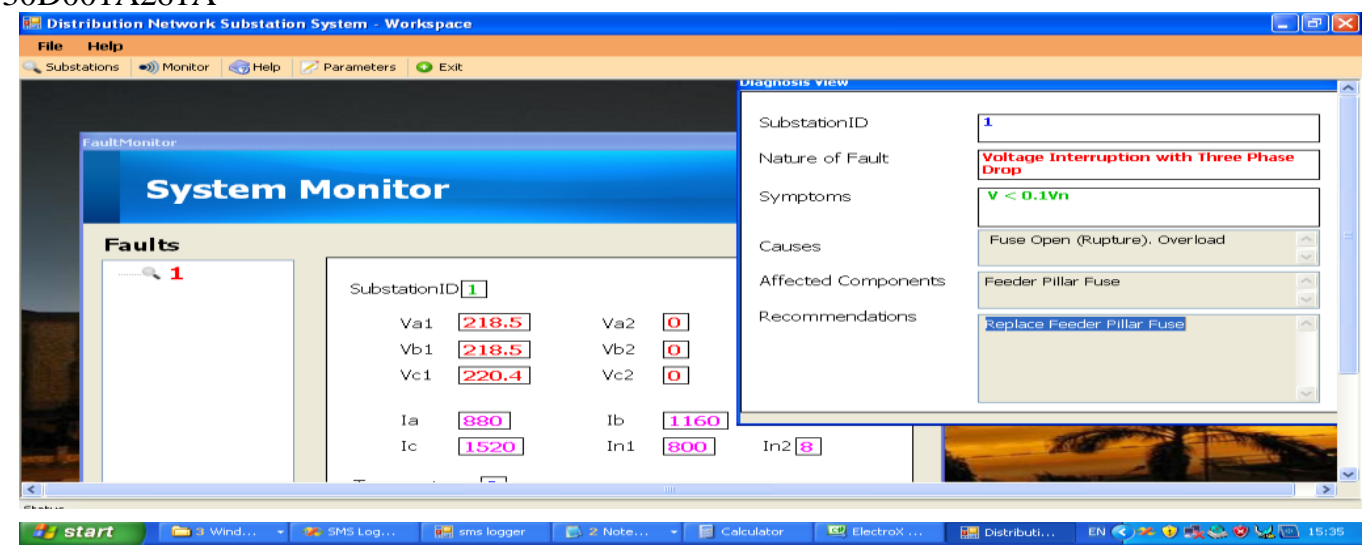

ReceivedData

Figure 7:Three Phase Interruption

\$1001737374737300161D2614080000000000000000000000000000000C120C000F160F000D130D00141D14 000D130D001A261A

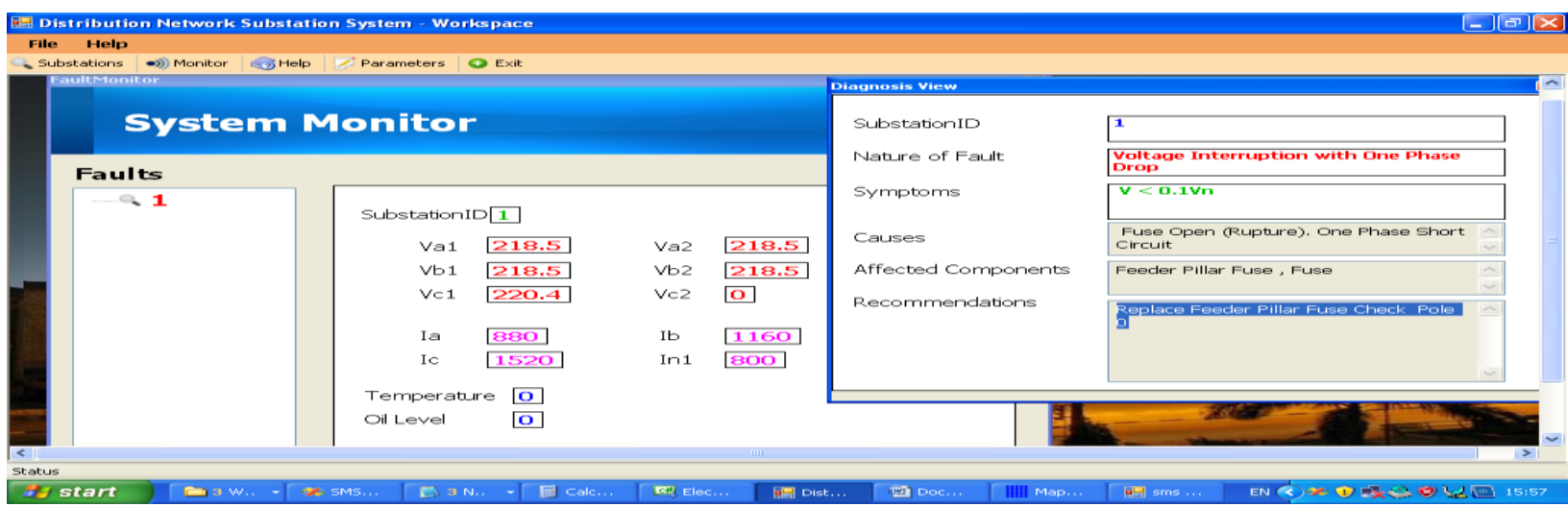

Figure 8: Interruption detection on one phase/Voltage Unbalance

Case 2: Received Data for Voltage unbalance

\$1001737374730000161D2614080000000000000000000000000000000C120C000F160F000D130D00141D14 000D130D001A261A 


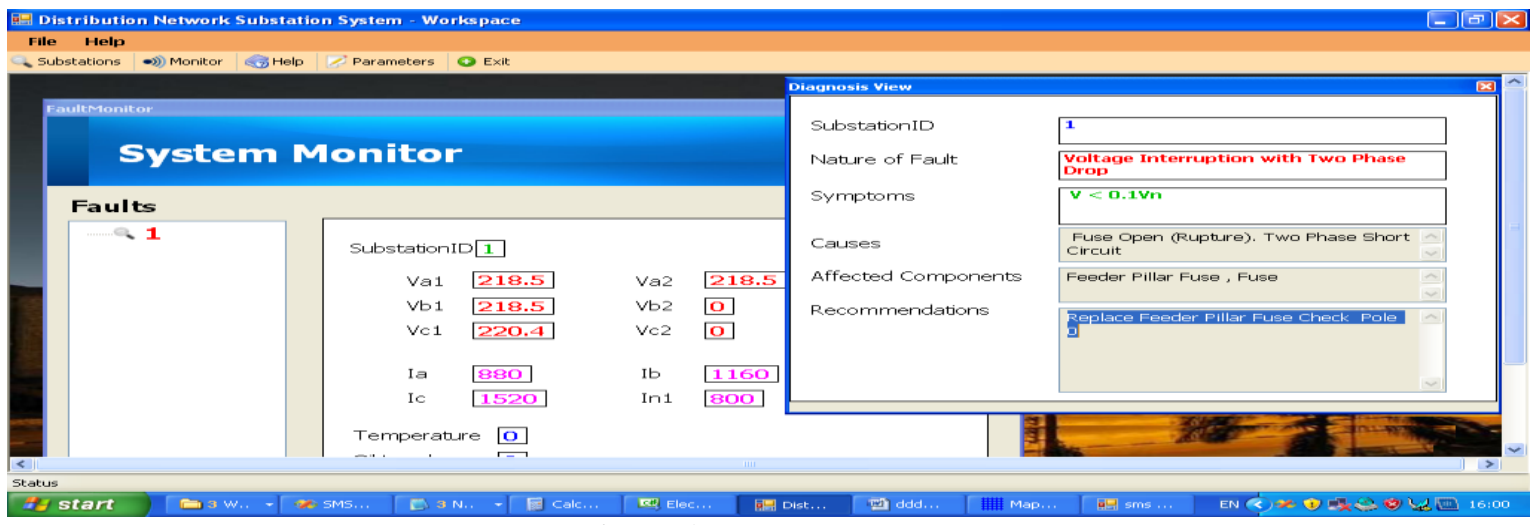

Figure 9: Voltage Unbalance

Case three: Received Data for Voltage swell:

\$1001737373737373001D2614080000000000000000000000000000000C120C000F160F000D130D00141D14 000D130D001A261A

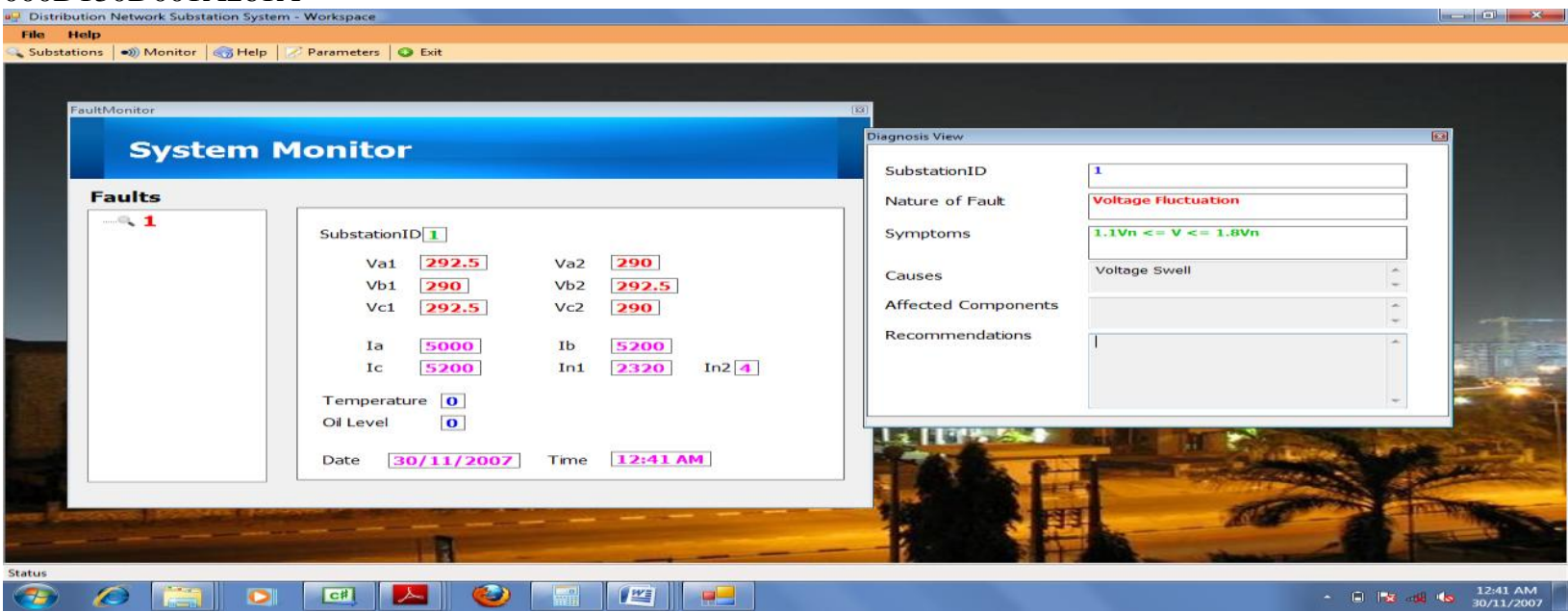

\section{CaseData}

Fig, 10 Voltage Swell

\$1001000000000000161D2614080000000000000000000000000000000C120C000F160F000D130D00141D14 000D130D001A261A

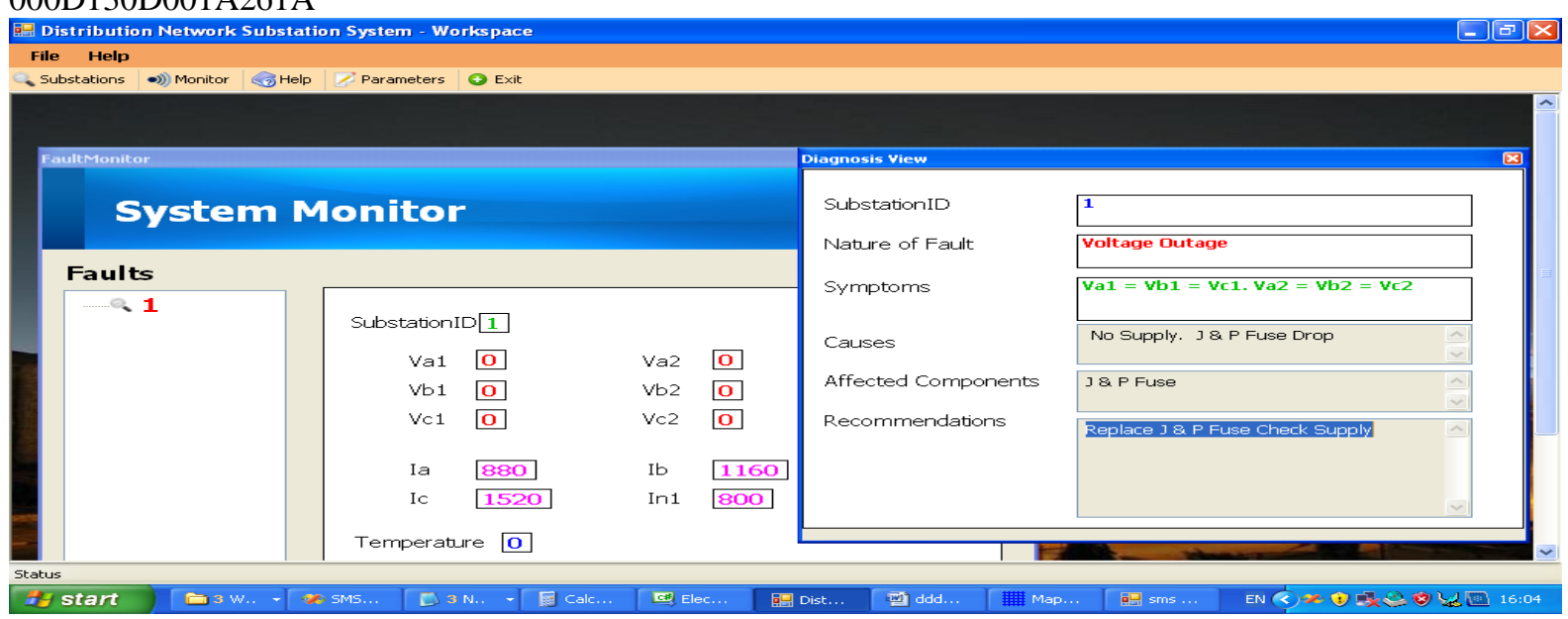

Figure 11: Long Duration Interruption 


\section{Adaptability Of Distribution Automation System To Electric Power Quality Monitoring In Nigeria}

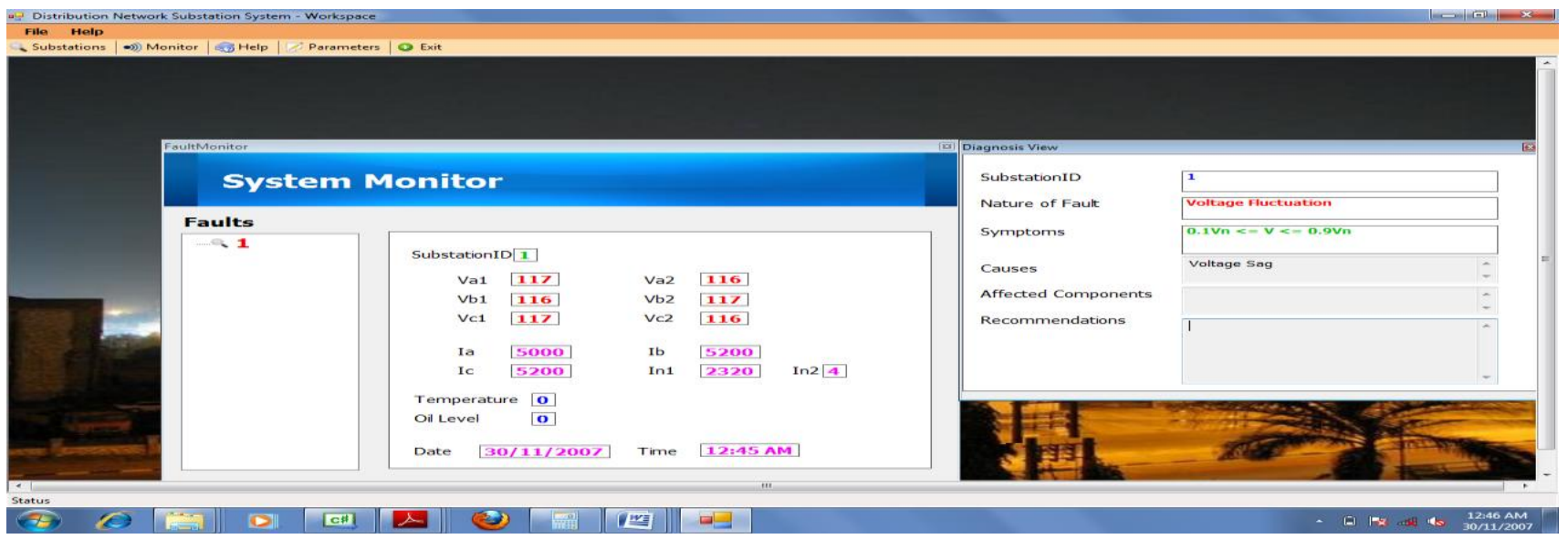

Fig. 12 voltage sag

\section{Conclusion}

Real-time monitoring of power quality is presented in this work. It was observed that the GSM-based DAS provides useful information that when analysed serves as basis for assessing the quality of supply. This was successfully implemented for Akure EPDS. The results shows that sustained interruptions and under voltage characterized Nigeria Electric Power Distribution System as observed from the record obtain in Akure EPDS investigated. This approach of determining the power supply quality provides platform of quality assesmentment in real-time at the substation level.

\section{References}

[1] W. Barry, Kennedy “power Quality Primer," McGraw-Hill, USA, (2000), 33-49

[2] H.A. Boknam S., PARK C., SHIN S. ,KWON and S.PARK Power Quality Monitoring on Distribution Network using Distribution Automation System, 19th International Conference on Electricity Distribution Vienna, (2007), Paper 0426

[3] Jurado, F.; Acero, N.; Ogayar, B. (2002), "Application of signal processing tools for power quality analysis"; Canadian Conference on Electrical and Computer Engineering, IEEE CCECE 2002. Vol. 1, 12 - 15

[4] Umar N K, (2007) Signal Processing Techniques used in Power Quality Monitoring Faculty of Electrical Engineering, Wroclaw University of Technology, Poland

[5] Styvaktakis E., Bollen M. H. J., Gu I.Y.H, (2002), "Automatic classification of power system events using RMS voltage measurements", IEEE Power Engineering Society Summer Meeting, vol.2, pp. 824- 829

[6] KEPRI "Final report of the development of optimal system in distribution network based on distribution automation," KEPCO, Korea, pg 193-202, 2005

[7] Bollen M. H. J (1999), Understanding power quality problems, Voltage sags and interruptions, New Jersey, USA, IEEE Press, , pp 541.

Brand K., Lohmann V., and Wimmer W. (2003), "Substation Automation Handbook”, Utility Automation Consulting Lohmann, Bremgaten, Switzerland,

[8] Daponte P, Penta M. , Mercurio G. (2004), "TRANSIENTMETER: A Distributed Measurement System for Power Quality Monitoring", IEEE Transactions on Power Delivery, Vol. 19, pp: 456-463,

[9] Nigeria Communication Website, www.ncc.nig.com, $24^{\text {th }}$ June, 2008

[10] R. P Gupta Distribution Automation System, Indian Institute of Technology Gupta Kanpur -pg16 2008

[11] IEC 61850, "Communication Network and System in Substation", "Communication Networks and System in Substation", Part 1, IEC 61850, 2003.

[12] R.P Gupta, M. Pandey and N. Srivastava, "Data Communication Architecture using IEC 61850 Protocol for Substation Automation", in the proceedings of International Conference on Distribution India, pp 113-121, 2004

[13] R. P. Gupta, and S. C. Srivastava, "Technology Development and Implementation for Power Distribution Automation", Water and Energy International Journal, Vol. 61, No. 4, pp. 40-47, 2004

[14] N. James, "Control and automation of electrical power distribution systems", CRC press Boca Raton, , pg292, 2007

[15] D. Estra1da, I.I. Maria and S.A.R. Macau, "China Distribution Automation over Power Line Carrier Communication System" in Macau 2008

[16] McDonald J. D., "Substation Automation: IED Integration and Availability of Information", IEEE Power and Energy Magazine, pp. 22-31, 2003.

[17] Tenbohlen S, Figel F., (2000), “On-line Condition Monitoring for Power Transformers”, IEEE Power Engineering Society Winter Meeting, Singapore, 